PALEO

Revue d'archéologie préhistorique

Numéro spécial | 2009-2010

Entre le marteau et l'enclume...

\title{
La question du débitage de grands éclats à l'Acheuléen
}

The question of Acheulean large flakes debitage

\section{Vincent Mourre et David Colonge}

\section{(2) OpenEdition}

\section{Journals}

Édition électronique

URL : http://journals.openedition.org/paleo/1920

DOI : $10.4000 /$ paleo.1920

ISSN : 2101-0420

Éditeur

SAMRA

Édition imprimée

Pagination : $35-48$

ISSN : 1145-3370

Référence électronique

Vincent Mourre et David Colonge, "La question du débitage de grands éclats à l'Acheuléen », PALEO

[En ligne], Numéro spécial | 2009-2010, mis en ligne le 23 avril 2012, consulté le 07 juillet 2020. URL

http://journals.openedition.org/paleo/1920 ; DOI : https://doi.org/10.4000/paleo.1920

\section{(c) (i) (9)}

PALEO est mis à disposition selon les termes de la licence Creative Commons Attribution - Pas d'Utilisation Commerciale - Pas de Modification 4.0 International. 


\title{
LA QUESTION DU DÉBITAGE DE GRANDS ÉCLATS À L'ACHEULÉEN
}

\author{
Vincent MOURRE ${ }^{(1)}$ et David COLONGE ${ }^{(2)}$
}

Résumé : Dès ses origines, peut-être plus encore que par les bifaces, l'Acheuléen africain est caractérisé par le débitage d'éclats de grands modules, pouvant atteindre plusieurs kilos et plusieurs dizaines de centimètres. Ces éclats ont été mis à profit pour la réalisation de différents outils, dont des bifaces et des hachereaux, mais ils ont également pu être exploités comme nucléus. La fréquence des grands éclats caractérise également l'Acheuléen dans la plupart des régions où il est connu, à de notables exceptions près telles que l'Europe nord-occidentale.

Un intérêt inégal a été porté à la question des techniques employées pour produire de grands éclats : éludée par certains auteurs, évacuée sommairement par d'autres, elle a aussi passionné des préhistoriens tels que H. Breuil, qui a proposé des hypothèses peu réalistes faisant intervenir des systèmes complexes de trépieds et de balanciers.

L'hypothèse la plus courante fait intervenir la percussion directe d'un nucléus sur un percuteur dormant immobilisé au sol. Un angle d'éclatement très ouvert (jusqu'à $170^{\circ}$ ) et des cônes de percussion multiples sont traditionnellement considérés comme caractéristiques de cette technique, parfois qualifiée improprement de "débitage sur enclume ", de " débitage bloc contre bloc » ou de " technique clactonienne».

Des données expérimentales et des exemples archéologiques fournis par différents sites acheuléens incitent à relativiser l'importance de cette technique et conduisent à proposer des techniques alternatives moins spectaculaires mais qui rendent plus fidèlement compte des caractéristiques des grands éclats de l'Acheuléen et des nucléus dont ils sont issus.

Mots-clés : Acheuléen, techniques de débitage, débitage sur percuteur dormant, nucléus.

Abstract: The question of Acheulean large flakes debitage. As of its origins, perhaps even more than by bifaces, African Acheulean is characterized by the flaking of large flakes, which can reach several kilos and several tens of centimeters. These flakes were used for the realization of various tools, of which bifaces and cleavers, but they also could be exploited as cores. The frequency of the large flakes also characterizes Acheulean in the majority of the areas where it is known, with notable exceptions such as northwestern Europe.

An unequal interest was carried to the question of the techniques employed to produce large flakes: ignored by certain authors, summarily shrugged off by others, it also impassioned scholars such as $\mathrm{H}$. Breuil, which proposed not very realistic assumptions involving complex systems of tripods and pendulum.

The most current assumption involves direct percussion of a core on a resting hammer immobilized on the ground. A very wide flaking angle (up to $170^{\circ}$ ) and multiple cones of percussion are traditionally regarded as characteristics of this technique, sometimes called improperly «anvil chipping technique», "block on block» or " clactonian technique».

Experimental data and archaeological examples provided by several Acheulean sites lead to revise the importance of this technique and result in proposing less spectacular alternative techniques but which more accurately account for the characteristics of the large acheulean flakes and of the cores from which they were struck.

Key-words: Acheulean, flaking techniques, flaking on resting hammer, core.

(1) INRAP et TRACES/UTAH UMR 5608 du CNRS - Les Haut Arthèmes, 84560 Ménerbes - vincent.mourre@inrap.fr

(2) INRAP et TRACES/UTAH UMR 5608 du CNRS - Dardenne, 46300 Le Vigan - david.colonge@inrap.fr 


\section{ACHEULÉEN ET GRANDS ÉCLATS}

La notion d'Acheuléen a été introduite à la fin du XIX siècle par G. de Mortillet suite à la découverte d'industries comportant de nombreux bifaces dans les alluvions de la moyenne terrasse de la Somme, à Saint-Acheul près d'Amiens. Par extension, le terme "Acheuléen " a été appliqué à des industries à bifaces qui font leur apparition en Afrique vers 1,6 Ma BP et qui se développent par la suite dans tout l'Ancien Monde, en particulier en Europe entre environ 600000 et 200000 ans BP (Tuffreau 1996).

La présence de bifaces est donc l'élément le plus consensuel de la définition de l'Acheuléen, même si certains auteurs estiment qu'il convient de tenir compte de la fréquence de ces pièces au sein de l'outillage avant d'attribuer celle-ci à ce technocomplexe : M. Kleindienst (1961, p. 40) puis M. Leakey (1975, p. 481) considèrent ainsi qu'un taux minimum de $40 \%$ de bifaces est nécessaire pour considérer qu'une série est acheuléenne. François Bordes rejetait ce critère et estimait qu' ' $A ̀$ ce compte-là, il n'y aurait sans doute pas d'Acheuléen à Saint-Acheul. " (Bordes 1984, p. 217). Peut-être plus encore que par les bifaces, l'Acheuléen africain est caractérisé dès ses débuts par le débitage d'éclats de grandes dimensions, dépassant fréquemment $150 \mathrm{~mm}$ : le site EF-HR, au sommet du Bed II d'Olduvai en Tanzanie a notamment livré de grandes pièces bifaciales réalisées sur éclat, dont un certain nombre de hachereaux parmi les plus anciens connus (1,6 Ma ?) (Leakey 1971) (fig. 1a).

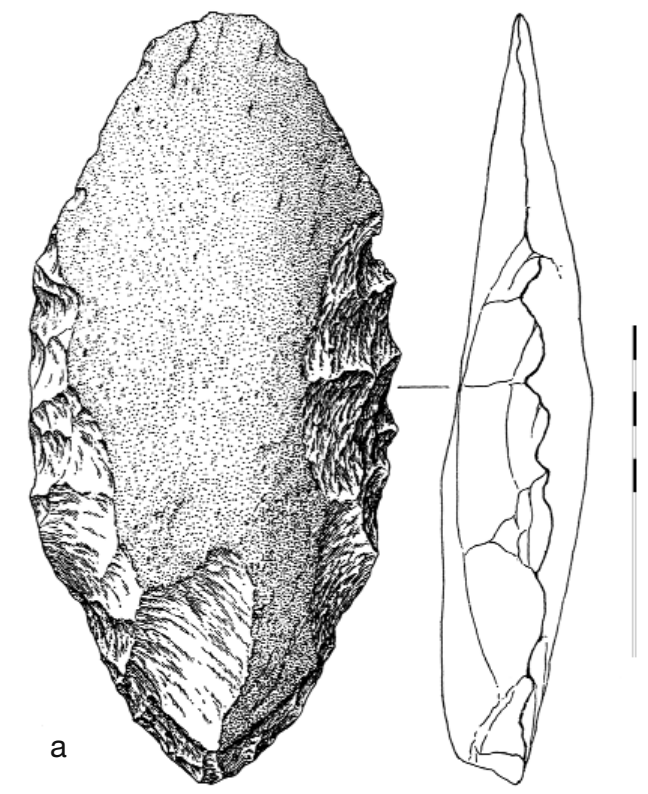

Certains sites acheuléens plus récents (Pléistocène moyen) ont également livré des outils spectaculaires. Un hachereau de Bangué I, en République centrafricaine, présente des dimensions particulièrement imposantes : « II s'agit d'un hachereau en quartzite mesurant 0,265 $\mathrm{m}$ de long, 0,180 $\mathrm{m}$ de largeur et pesant près de $4 \mathrm{~kg}$. (...) [De tels] objets posent le problème du débitage car leur fabrication a demandé un éclat de très grandes dimensions qui n'a pu être obtenu que par percussion sur percuteur dormant. » (Bayle des Hermens 1975 ; pp. 71-72) (fig. 1b). De même, un hachereau de l'Erg Tihodaïne (Algérie) atteint les dimensions exceptionnelles de $280 \times 190 \times 64 \mathrm{~mm}$ pour $3405 \mathrm{~g}$ (Mourre 2003). Clarence Van Riet Lowe mentionne un éclat de 14,4 kg et un biface sur éclat de 3,6 kg à Isimila en Tanzanie (Van Riet Lowe 1951). Bernard Champault signale à Tachenghit des hachereaux sur éclats Kombewa atteignant $1 \mathrm{~kg}$, eux-mêmes issus d'éclats-nucléus dont la masse est estimée à $6 \mathrm{~kg}$ (Champault 1966, p. 322 sqq.). Hors d'Afrique, les grands éclats sont fréquents dans la plupart des sites acheuléens, notamment transformés en hachereaux. Quelques régions semblent faire exception, comme l'Europe nord occidentale même si quelques hachereaux sont connus au Royaume-Uni (Mourre 2003).

II paraît légitime de tenter de déterminer quelle(s) technique(s) de percussion a (ont) été employée(s) pour débiter les « grands éclats » au cours de l'Acheuléen, et ce pour plusieurs raisons. II s'agit avant tout de contribuer à reconstituer l'histoire générale des techniques, ce que certains jugeront peut-être anecdotique, mais il s'agit également d'aborder la question des capacités conceptuelles et motrices des anciens Hominidés. La question de la technique employée pour débiter les grands éclats acheuléens a connu un intérêt très inégal au cours du temps.

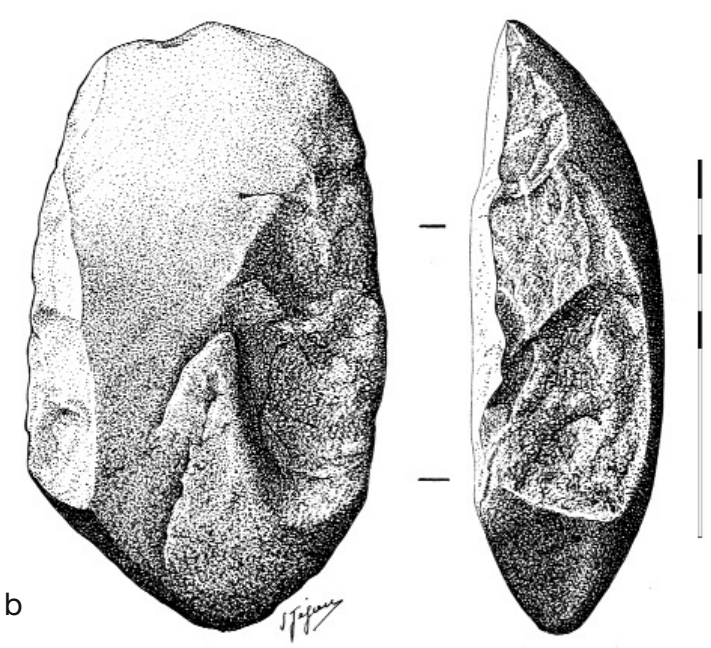

Figure 1 - Outils acheuléens sur grands éclats. a : site EF-HR, Bed II d'Olduvai, Tanzanie (d'après Leakey 1971) ; b : site de Ngoéré-Ngolo, République centrafricaine (d'après Bayle des Hermens 1975) ; (échelles $=10 \mathrm{~cm}$ ).

Figure 1 - Acheulean tools made from large flakes. a : EF-HR site, Bed II of Olduvai, Tanzania (after Leakey 1971) ; b : Ngoéré-Ngolo site, Central African Republic (after Bayle des Hermens 1975); (scale bars $=10 \mathrm{~cm}$ ). 
Paradoxalement, elle semble avoir plus passionné les

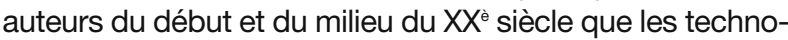
logues expérimentateurs modernes, ceux-ci considérant peut-être le problème comme définitivement résolu par ceux-là. Les études expérimentales consacrées à la production d'outils bifaciaux acheuléens se focalisent souvent sur les ultimes phases du schéma de production, à savoir le façonnage des outils, délaissant les phases initiales de production des éclats supports. Certains expérimentateurs actuels ont d'ailleurs façonné des bifaces et des hachereaux aux dépens de grands éclats débités à la masse métallique.

Deux principales hypothèses concurrentes sont envisagées dans la littérature pour le débitage de grands éclats : l'une, que nous écarterons assez rapidement, fait intervenir un appareillage complexe basé sur le principe du balancier; l'autre, plus traditionnelle et plus répandue, fait appel à la percussion sur percuteur dormant.

\section{UNE HYPOTHÈSE OSÉE : LA PERCUSSION À L'AIDE D'UNE « MACHINE »}

Une hypothèse particulièrement osée et assez méconnue a été proposée par $\mathrm{H}$. Breuil. Elle fait intervenir un percuteur massif suspendu à un trépied et actionné comme un balancier.

L'idée est évoquée laconiquement dans le compte-rendu d'une communication faite à la Société préhistorique française : selon $\mathrm{H}$. Breuil, dans l'Acheuléen des rives de la rivière Vaal en Afrique du Sud, la taille de grands galets de dolérite "suppose des procédés d'une très grande puissance mécanique, capables de produire d'énormes éclats, retaillés ensuite en outils. La production de tels éclats, pouvant atteindre et dépasser 0m40, dépasse l'application d'une force de percussion manuelle ou sur enclume et suppose l'emploi, pour cela, de quelque technique mécanique plus compliquée. » (Breuil 1949a). L'auteur développe cette idée dans Beyond the bounds of History, ouvrage de vulgarisation richement illustré de sa main : "L'auteur a suggéré que la méthode employée soit le débitage par des coups donnés par un gros bloc accroché à un trépied. Ce bloc serait balancé vers la pièce à tailler, à la manière d'un pendule - une opération qui aurait nécessité les efforts combinés d'au moins trois personnes - deux pour régler le mouvement de la roche en oscillation et pour l'empêcher de frapper au retour, la troisième pour diriger et corriger la trajectoire, tandis qu'elle se protégeait des esquillements et du percuteur derrière une fourmilière ou un grand rocher. " (Breuil 1949b, p. 41) ${ }^{3}$ (fig. 2).

L'idée d'une utilisation de percuteurs mus par des balanciers a été reprise par D. Cahen (1975) puis par D. Muya (1991) à propos du débitage de grands éclats-supports à hachereaux du site acheuléen de La Kamoa (R.D. du Congo). L'hypothèse de $\mathrm{H}$. Breuil est également mentionnée par C. Van Riet Lowe (1951) et par B. Champault (1966, p. 336 sqq.). Elle nous semble peu probable, non que nous considérions a priori la mise en œuvre d'un tel mécanisme comme hors de portée des artisans acheuléens, mais parce qu'elle n'est pas la plus parcimonieuse et que les caractéristiques des éclats en question peuvent s'expliquer sans faire appel à l'emploi d'une technique aussi complexe et probablement délicate à utiliser.

\section{L'HYPOTHÈSE TRADITIONNELLE : LA PERCUSSION SUR PERCUTEUR DORMANT}

L'hypothèse la plus courante en ce qui concerne la technique de débitage des grands éclats acheuléens est l'utilisation de la percussion sur percuteur dormant : un nucléus mobile, tenu à une ou deux mains, vient heurter un percuteur immobile afin de détacher un éclat.

\section{Terminologie}

De nombreuses expressions ont été employées pour désigner cette méthode (notamment " technique sur enclume ", « technique clactonienne ", « technique bloc contre bloc ») et une certaine confusion règne dans la terminologie malgré différentes tentatives de mise au point. L'expression la plus adaptée, «percussion sur percuteur dormant ", a été vulgarisée par F. Bordes qui l'emploie en proposant malheureusement comme synonyme « percussion sur enclume »(Bordes 1961, p. 13).

Dès 1967, L. Balout notait : « (...) l'expression anglaise «block on block» et la française "débitage sur enclume» sont également vicieuses. La taille au percuteur manuel de pierre est aussi une taille "bloc contre bloc», et Fr. Bordes constate que les stigmates ne sont pas toujours aisés à distinguer de ceux d'une taille "sur enclume» (1961, p. 8). Celle-ci n'est vraisemblable que pour le débitage de gros éclats, en particulier des "éclats-nucléii utilisés à Ternifine et ailleurs pour produire des hachereaux très particuliers ; mais elle n'est pas nommée. On forge «entre enclume et marteau", ce qui n'est pas ici le cas. Fr. Bordes a proposé de remplacer "enclume» par "percuteur dormant», ce qui est très judicieux et devrait être généralement adopté. » (Balout 1967, p. 705).

La percussion sur percuteur dormant a très souvent été qualifiée de technique " clactonienne » du fait de la fréquence supposée de sa mise en oeuvre dans certaines industries «post-Abbevilliennes et pré-Acheuléennes » (Breuil 1954). Outre le fait que l'abandon de cette expression a fait l'objet d'une résolution lors du $1^{\text {er }}$ Congrès Panafricain de Préhistoire (citée par L. Balout 1967, p. 704),

(3) "The present writer has ventured to suppose that chipping by blows given by a big block hung on a tripod may have been the method. This block would be swung towards the piece to be split, in the manner of a pendulum - an operation that would need the combined efforts of at least three persons - two to regulate the movement of the swinging rock and to prevent it striking on the return journey, the third to direct and correct the trajectory, whilst being himself protected from the splinters and from the striking block by an ant-heap or a large boulder. » 


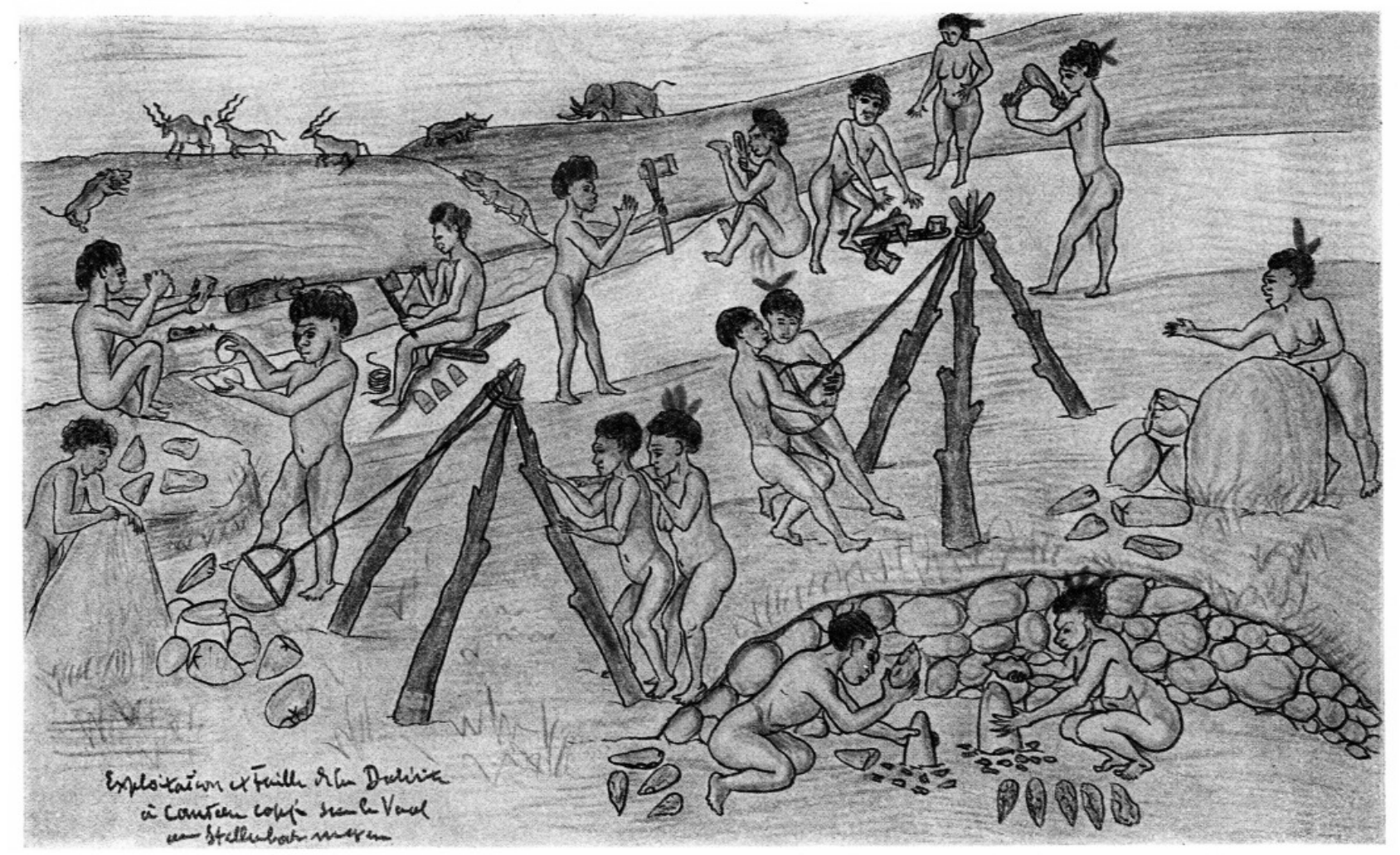

Figure 2 - Débitage de grands éclats à l'aide de trépieds vu par H. Breuil (1949). La légende manuscrite indique "Exploitation et taille de la dolérite à Canteen Copje sur le Vaal au Stellenbosch moyen ».

Figure 2 - Large flakes detachment using tripods as pictured by H. Breuil (1949). The handwritten caption mentions "Working and knapping of dolerite at Canteen Copje on the Vaal River during the Middle Stellenbosch ».

il semble opportun de n'appliquer le qualificatif « clactonien " qu'au schéma de production mis en évidence dans les industries paléolithiques du sud du Royaume-Uni, notamment à Barnham, Swanscombe et High Lodge (Ashton et al. 1992), et nommé Système par Surfaces de Débitage Alternées par H. Forestier (Forestier 1993). En tout état de cause, l'usage n'a pas retenu le terme « clactonien ", faisant référence à des éléments culturels ou chronologiques, pour désigner une technique.

Don E. Crabtree emploie indifféremment les expressions " anvil technique » et « block on block », qu'il définit ainsi : " méthode consistant à détacher des éclats en percutant le nucléus contre l'enclume (...). Peut être utilisé pour produire des éclats épais comme dans l'industrie clactonienne ou des lames à talons fins. " (Crabtree 1972, p. 48) ${ }^{4}$.

Alors que l'utilisation de la taille sur percuteur dormant est peu évoquée par les auteurs occidentaux modernes, elle a fait l'objet d'attentions continues en Chine depuis les travaux de Pei Wenzhong ${ }^{5}$ en 1936. La technique y est connue sous le nom de "Peng Zhen Fa ", littéralement
« méthode de percussion sur enclume » (Shen et Wang 2000).

\section{Descriptions}

L'une des premières descriptions de la technique de taille sur percuteur dormant semble être celle de Pei : « $M$. Coutier a fait souvent des démonstrations de taille de grès et silex sur enclume. En tenant un gros bloc de grès et en le laissant tomber, tout en dirigeant sa chute à une faible vitesse, sur la pointe d'un bloc dormant ou enclume, il a obtenu rapidement et en grand nombre de très grands et larges éclats, souvent assez longs, détachés du bloc mobile. Cette très grande dimension de l'éclat ne peut être obtenue par le procédé manuel (...) » (Pei 1936).

François Bordes en livre également une description en mentionnant les expériences de L. Coutier : «Ce procédé convient particulièrement à l'obtention de très grands éclats. On pose à terre une grosse pierre présentant une saillie qui va servir d'enclume [sic]. Le nucléus tenu à deux mains est brandi au-dessus de la tête, puis heurté vigoureusement sur l'enclume. » (Bordes 1947, p. 17).

(4) « method of removing flakes by swinging the core against an anvil (...). May be used to produce large thick flakes as in the Clactonian industry or blades with thin platforms. »

(5) anciennement transcrit Pei Wen Chung. 
$H$. Breuil et R. Lantier en donnent aussi une description assez proche : «Dans ce cas, on laisse tomber, en dirigeant la chute de manière à ce que le choc se produise à l'angle et au point voulu, le bloc à débiter sur la pointe de la pierre servant d'enclume, ou percuteur dormant. À la condition que la matière première se rencontre en blocs volumineux, ce procédé est très supérieur au premier [percussion directe classique] pour obtenir des éclats de très grandes dimensions, ce qui est absolument irréalisable par percussion manuelle. Cela représente également un bien moindre effort, puisque c'est en somme la pesanteur qui se substitue à l'impulsion de la main. » (Breuil et Lantier 1951, p. 6768). Henri Breuil a lui-même pratiqué cette technique, comme en témoigne une photographie de l'ouvrage Sur les chemins de la préhistoire : l'abbé Breuil du Périgord à l'Afrique du Sud (Coye dir. 2006, p. 163).

\section{Principes physiques}

Dans son étude des Théories physiques et mathématiques de la taille des outils préhistoriques (1989), H. Bertouille analyse comme suit la taille sur percuteur dormant : "Comme dans la taille à plan de frappe imposé [percussion " bipolaire " sur enclume], la taille sur enclume procède du choc de deux pièces dont l'une a une extrémité fixe. Mais ici, le nucléus sert en même temps de percuteur. En effet, dans ce système de taille, la pièce tenue en main (souvent à deux mains) est percutée violemment sur une proéminence d'une enclume dormante. Cette façon d'opérer à cause, en particulier, des conditions de préhension de la pièce, exige l'emploi de nuclei volumineux. La précision de l'impact est relativement mauvaise. En outre, surtout si l'enclume est posée à même le sol et l'artisan agenouillé, la protection des mains implique souvent que le nucléus heurte l'enclume obliquement par rapport à l'horizontale (...). La force $F$ appliquée est donc oblique par rapport au plan de frappe. » (Bertouille 1989, p. 43-44).

Les principales conclusions de l'auteur présentant un intérêt en ce qui concerne la technologie et la reconnaissance des stigmates sur le matériel archéologique sont les suivantes:

- « Le point d'impact se trouve en pleine pièce ou tout au moins assez loin de l'arête du nucléus. Les conditions de la rupture sont donc sensiblement celles d'un milieu semiinfini chargé ponctuellement, avec une des deux pièces en présence ayant une extrémité fixe. ”

- « Le point d'impact elliptique de hertz est bien marqué du fait de l'importance des masses en présence et de la grande force développée. »

- «Le bulbe de percussion tend vers la sphère parfaite de Boussinesq ; il est donc saillant et bien étendu. Le cône neutre qui sécante cette sphère y a par conséquent son maximum de développement. » (Bertouille 1989, p. 44).

\section{Percuteurs dormants archéologiques}

Un certain nombre de vestiges archéologiques considérés comme des percuteurs dormants potentiels viennent à l'appui de l'hypothèse de l'utilisation d'une telle technique à l'Acheuléen.
Marie-Henriette Alimen présente comme un percuteur dormant un volumineux bloc de quartzite $(25 \times 23 \times 13 \mathrm{~cm}$; $15,6 \mathrm{~kg}$ ) provenant de l'Acheuléen supérieur de l'Oued Farès, dans le Sahara occidental algérien (Alimen 1963 ; Alimen 1978, pp. 272-273). L'une des spécificités de cet objet est qu'il aurait été aménagé : un certain nombre d'éclats de façonnage en auraient été détachés afin de dégager un robuste éperon formant la touche du futur percuteur dormant.

Le site acheuléen moyen d'En Jacca - La Sauvegarde, près de Toulouse, a également livré un volumineux galet de quartzite pyrénéen $(27 \times 18 \times 16 \mathrm{~cm}$ ) et présentant de nombreux points d'impact plaidant en faveur de son interprétation comme percuteur dormant (Capdeville et al. 1997).

\section{Caractéristiques des produits}

Les descriptions des caractéristiques des éclats produits par taille sur percuteur dormant sont quasiment identiques chez différents auteurs :

- « Le plan de frappe [sic] est toujours grand, souvent lisse et très oblique par rapport à la face d'éclatement; l'angle entre ces deux plans atteint de $90^{\circ}$ à $170^{\circ}$. Sur le plan de frappe, le point d'impact est souvent marqué par une ou plusieurs fentes circulaires (bulbes incipients d'éclatements manqués). (...) Le bulbe est toujours gros ; il présente souvent des bulbes jumeaux contigus, chacun avec un cône grand et bien marqué. (...) La face d'éclatement présente parfois de très fortes ondulations. " (Pei 1936, pp. 52-53). - «Dans les éclats obtenus sur enclume, le plan de frappe [sic] est lisse, très oblique par rapport au plan d'éclatement parfois jusqu'à $170^{\circ}$; celui-ci, naturel ou obtenu par une première taille, montre fréquemment des impacts réitérés dus à des chocs n'ayant pas abouti à l'éclatement désiré. Le cône et le bulbe au centre du plan de frappe sont très développés. Le premier est toujours bien formé, souvent à demi entouré de menus éclatements à son pourtour, allant parfois jusqu'à le dégager entièrement en éclatant tout le plan de frappe. Ce cône est souvent double ou triple, soit que des cônes incipients antérieurs, noyés dans la masse du silex, aient été mis à nu, soit que la percussion ait porté sur plusieurs saillies de l'enclume à la fois. Ce cône est le centre des nombreuses carènes, écaillures et esquilles, formant tout autour une gerbe en éventail. » (Breuil et Lantier 1951, p. 68).

- "Les éclats détachés présentent un très large plan de frappe [sic], très oblique, avec un gros conchoïde de percussion, un point d'impact bien visible, et un cône très apparent, parfois multiple. Si en effet l'enclume présente deux saillies rapprochées qui sont heurtées simultanément par le nucléus, chacune d'elles joue le rôle de percuteur. II y aura donc deux cônes au lieu d'un. " (Bordes 1947, p. 17). Toutefois, le même auteur nuancera par la suite sa description : « (...) la taille au percuteur dormant peut parfois produire accidentellement des éclats sans talon ni conchoïde, et ceci plus fréquemment que les autres techniques de débitage! » (Bordes 1961, p. 25). Dans le même ouvrage, il ajoute : "Nous mettons au défi [...] de prouver que tel éclat a été taillé bloc contre bloc plutôt qu'au percuteur mobile. Il ne s'agit pas de nier l'existence de la technique 
"sur enclume», mais elle est bien moins caractéristique qu'on ne l'a dit. » (Bordes 1961, note 2 p. 86).

Cette technique est souvent présentée comme la seule susceptible de fournir de grands éclats notamment pour la confection de hachereaux : aux auteurs précédents, il convient d'ajouter J. Tixier, du moins dans l'article fondateur consacré aux hachereaux nord-africains (Tixier 1956), J. Heinzelin de Braucourt (1962), M. Stekelis (1960) et D. Gilead (1973). L'hypothèse d'un détachement des grands éclats acheuléens par une percussion manuelle classique semble avoir été considérée plus ou moins implicitement comme peu vraisemblable pendant la deuxième moitié du XXè siècle.

\section{APPROCHE EXPÉRIMENTALE DE L'INDUSTRIE ACHEULÉENNE DE LANNE-DARRÉ}

Dans le cadre de l'étude de l'industrie lithique du site acheuléen de Lanne-Darré, nous avons mis en œuvre une approche expérimentale destinée à tester les deux hypothèses les plus vraisemblables concernant la production de grands éclats : laissant à de plus audacieux expérimentateurs le soin de tester la percussion au balancier, des éclats ont été produits par percussion sur percuteur dormant et par percussion manuelle, le percuteur étant tenu en main ou projeté sur un nucléus posé au sol.

\section{Présentation du site et de l'industrie lithique}

Lanne-Darré est un site acheuléen de plein air situé dans le piémont des Pyrénées centrales françaises (Colonge 2005 ; Colonge et Texier 2005). II s'étend sur une surface estimée à $6000 \mathrm{~m}^{2}$, dont 250 ont été explorés et 75 fouillés manuellement entre 1997 et 2003. Deux niveaux archéologiques ne constituant vraisemblablement qu'un seul ensemble plus ou moins résidualisé ont été identifiés à la base d'un important complexe colluvial d'une puissance actuelle de $2 \mathrm{~m}$. Celui-ci repose en contact discordant sur la Formation inférieure de Lannemezan, d'âge ponto-pliocène. Si l'industrie acheuléenne est en position secondaire, différents arguments liés à la paléo-topographie et aux états de surface indiquent une origine commune dans une aire limitée. Aucune tentative de datation absolue n'a abouti à ce jour.

Les matières premières utilisées sont essentiellement locales, même si une analyse fine révèle l'exploitation de sources diverses et complémentaires (Colonge et Mourre 2009 ; Colonge et al. à paraître). Les quartzites dominent largement le spectre des matériaux exploités (92 \%). Certains sont strictement locaux (66\%) et ont été utilisés dans toutes les phases des différentes chaînes opératoires ; d'autres ont été prélevés dans les cours d'eau proches ( $<2 \mathrm{~km} ; 32 \%)$ et sont représentés par des percuteurs, des nucléus et de grands éclats ; enfin, quelques hachereaux ont été réalisés aux dépens de quartzites provenant de cours d'eau plus éloignés (10 km ; $1 \%)$. Quelques petits fragments et outils en silex (1\%) proviennent de gîtes distants de 25 à 100 km.
L'industrie lithique de Lanne-Darré compte environ 3000 vestiges. Elle correspond essentiellement à une production de petits éclats par des méthodes variées dominées par le débitage Discoïde, associée à un outillage lourd dominé par les pièces bifaciales sur grands éclats. Les techniques mises en œuvre sont diverses : à la percussion directe au percuteur dur de petits nucléus ou d'outils façonnés tenus en main, il faut ajouter la percussion directe au percuteur tendre organique, utilisée de manière limitée mais sans équivoque pour le façonnage de quelques pièces bifaciales aux bords réguliers.

De manière relativement exceptionnelle pour l'Acheuléen, la présence à Lanne-Darré de plusieurs nucléus de grandes dimensions permet de documenter directement les modalités de production des grands éclats. Le plus important mesure $34,7 \times 24,5 \times 20,8 \mathrm{~cm}$ pour une masse de $28 \mathrm{~kg}$; il comporte deux surfaces taillées se recoupant à angle droit et présentant des séries d'enlèvements unipolaires (fig. 3a). Deux autres nucléus volumineux (fig. 3b : 23,6 $x$ $20,9 \times 16,8 \mathrm{~cm}$ pour $11,2 \mathrm{~kg}$; fig. $3 \mathrm{c}: 19,1 \times 24,7 \times 14,9 \mathrm{~cm}$ pour $9,5 \mathrm{~kg}$ ) présentent une importante série unipolaire d'enlèvements partant d'un plan de frappe néocortical. Ces nucléus portent des traces d'impact sur les faces opposées aux surfaces de plan de frappe. Le débitage des grands éclats exclusivement par percussion sur percuteur dormant semble donc a priori peu probable : d'une part, la masse et la morphologie des nucléus en question rend peu vraisemblable leur manipulation et, d'autre part, les stigmates évoqués précédemment évoquent des contrecoups liés à leur percussion alors qu'ils étaient calés sur des éléments en matières dures minérales.

\section{Grands éclats archéologiques et expérimentaux}

Comme évoqué précédemment, un talon lisse, des points d'impacts multiples, un cône et un bulbe très développés, parfois associés à de fortes ondulations sur la face inférieure, un angle d'éclatement très ouvert, pouvant atteindre $170^{\circ}$, sont classiquement considérés comme caractéristiques des éclats débités sur percuteur dormant. La plupart de ces critères ne peuvent pas être considérés comme diagnostiques : les talons lisses ne sont évidemment pas liés à une technique plutôt qu'une autre ; la multiplicité des points d'impact est liée à la nécessité de percuter plusieurs fois de suite avant le détachement d'un éclat et peut s'envisager dans le cas d'une percussion classique ; la présence de fortes ondulations est liée à une percussion particulièrement violente, pas à son mode d'application. En définitive, seul l'angle d'éclatement des éclats pourrait constituer un caractère objectif et diagnostique du débitage sur percuteur dormant s'il s'avérait que les résultats obtenus avec cette technique étaient significativement distincts de ceux obtenus par percussion classique.

Afin de tester la pertinence de ce critère, nous avons débité de grands éclats de quartzite par percussion sur percuteur dormant. Les angles de détachement obtenus sont présentés dans les histogrammes ci-dessous (fig. 4a). Ils sont comparés aux angles de détachement mesurés sur des éclats obtenus par percussion de blocs posés ou immobi- 

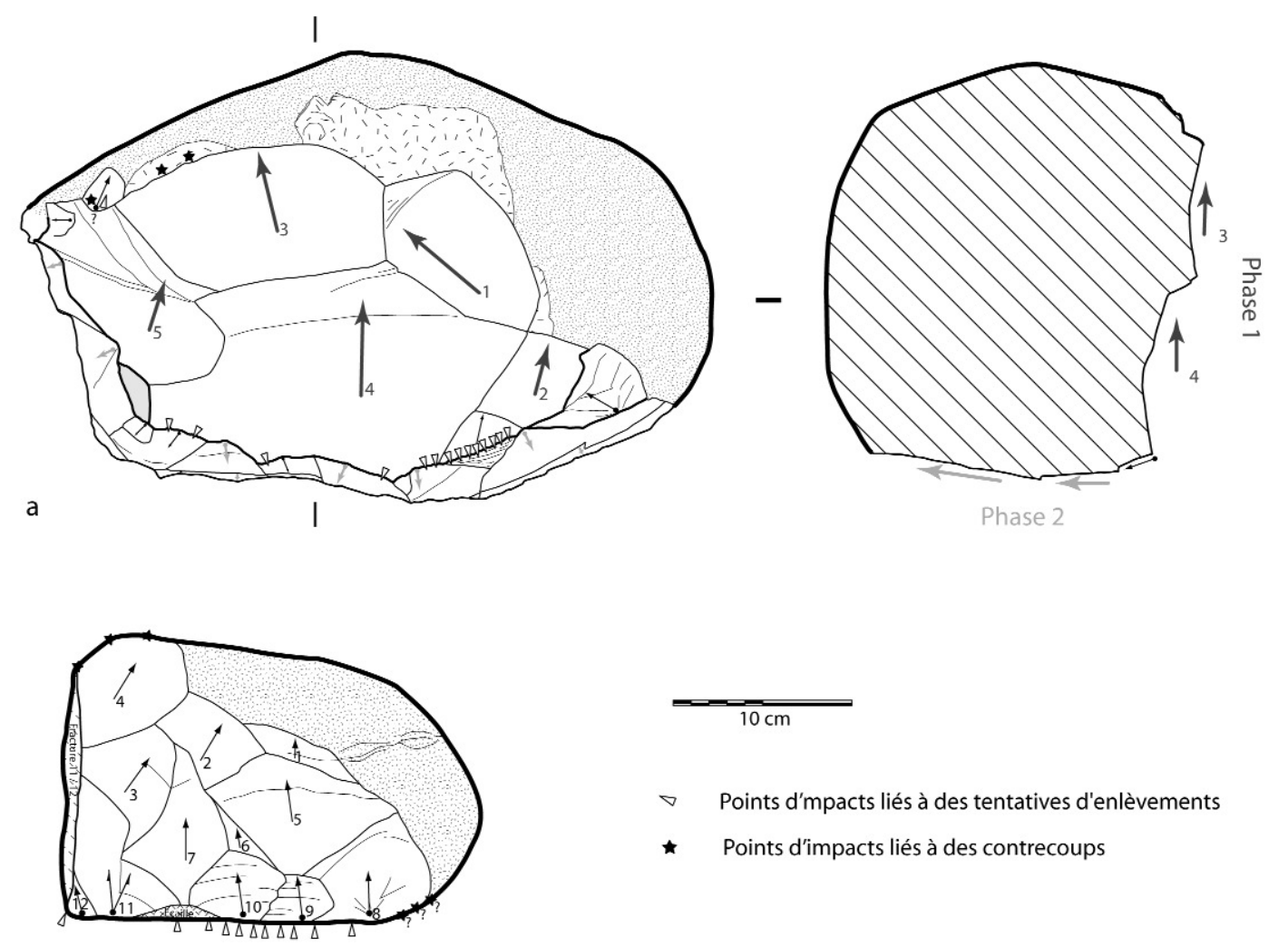

$\nabla \quad$ Points d'mpacts liés à des tentatives d'enlèvements

• Points d'impacts liés à des contrecoups

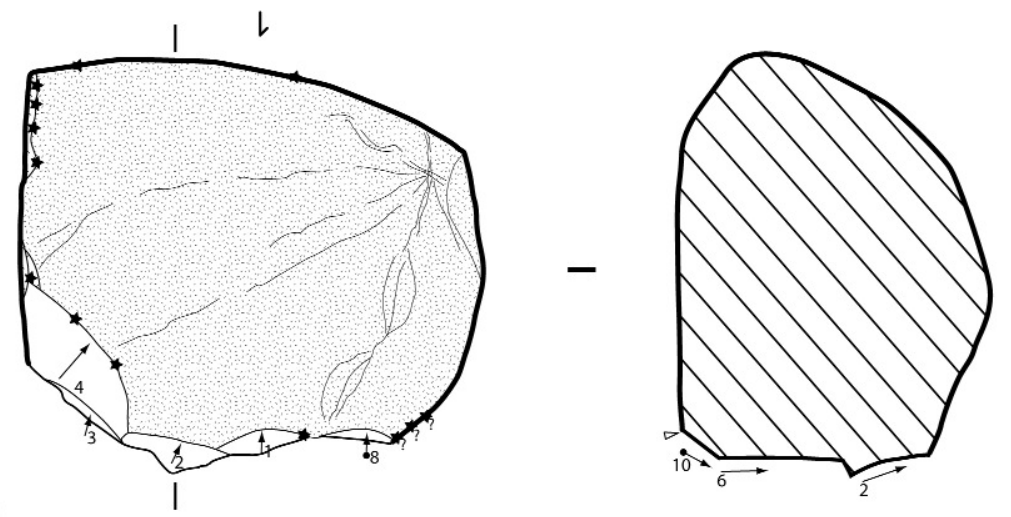

b

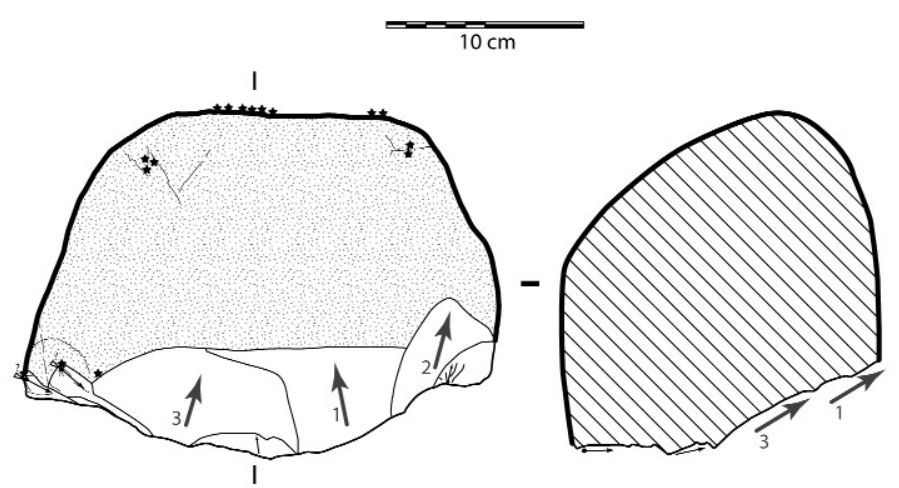

Figure 3 - Nucléus à grands éclats en quartzite de Lanne-Darré, Hautes-Pyrénées (DAO D.C.).

Figure 3 - Quartzite large flakes cores from Lanne-Darré site, Hautes-Pyrénées, France (CAD by D.C.). 
lisés au sol (fig. 4b) et sur les hachereaux de Lanne-Darré ayant encore leur talon (fig. 4c). L'angle moyen obtenu par percussion sur percuteur dormant $\left(110,2 \pm 8,6^{\circ}\right)$ est supérieur à celui obtenu par percussion directe classique (104,2 $\pm 6,3^{\circ}$ ) et légèrement inférieur à l'angle de détachement moyen des hachereaux de Lanne-Darré $(112,9 \pm 9,4)$. Les différences constatées sont toutefois limitées et les angles de détachement obtenus par percussion sur percuteur dormant sont tous inférieurs ou égaux à $130^{\circ}$, soit bien moins que les $170^{\circ}$ annoncés dans certaines publications.
Shen et Wang ont obtenu des résultats expérimentaux proches : «Un angle d'éclatement supérieur à $120^{\circ}$ a été considéré comme l'un des traits distinctifs du débitage sur percuteur dormant. Nos données montrent que l'angle d'éclatement moyen des 103 éclats observés est de $105^{\circ}$ $\pm 12,5$, avec une variation entre $71^{\circ}$ et $133^{\circ}$. Aucun des talons observés ne présente un angle supérieur à $140^{\circ}$, et environ la moitié des angles d'éclatement est compris entre 100 et $120^{\circ}(n=55)$ » (Shen et Wang 2000, p. 89) ${ }^{6}$ (fig. 4d). Ces auteurs ont également testé l'éventuelle corrélation
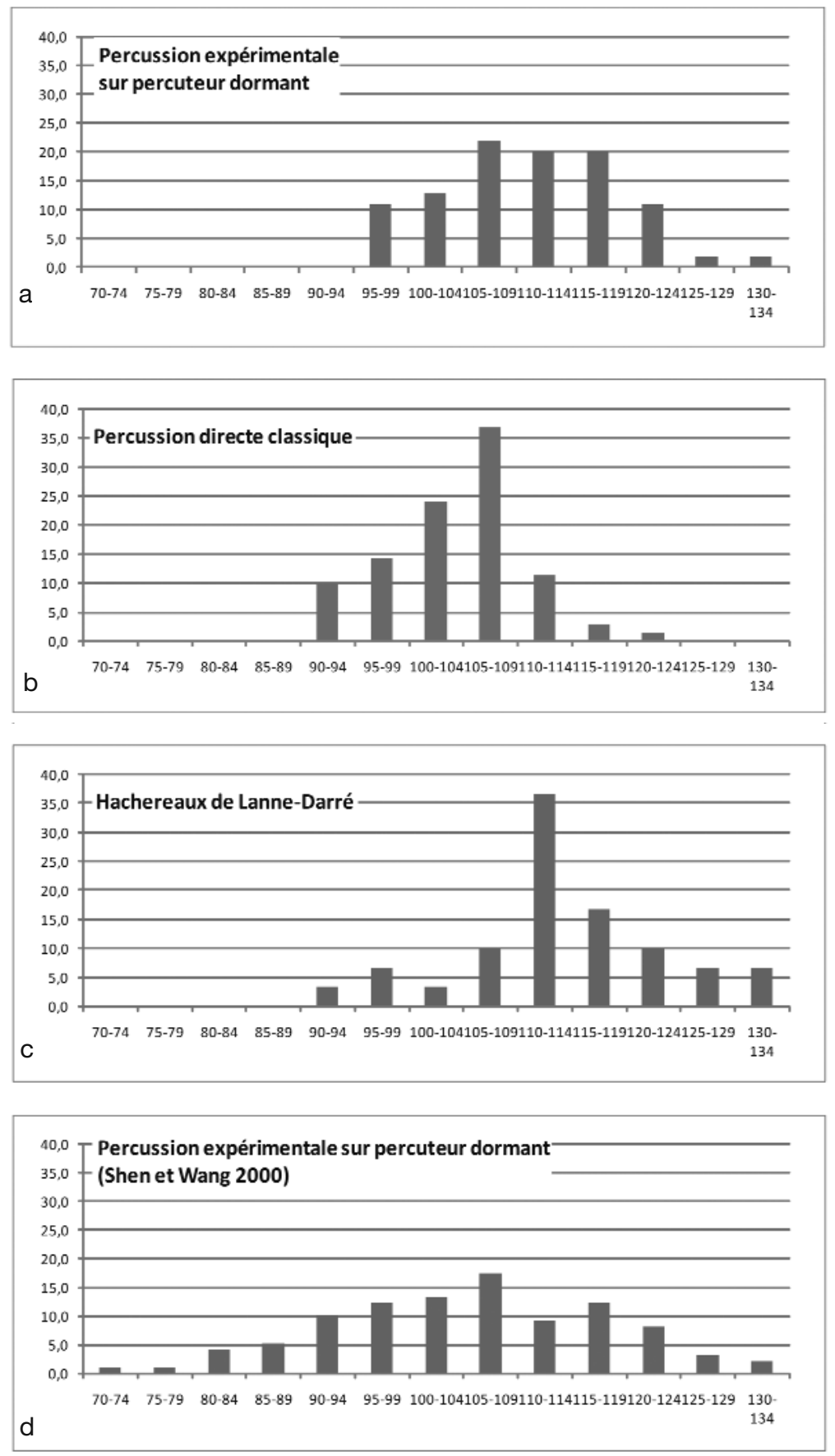

Figure 4 - Répartition par classes de 5 degrés des angles d'éclatements de grands éclats expérimentaux et archéologiques (pourcentages).

Figure 4 - Breakdown of the flaking angles of experimental and archaeological large flakes (classes of 5 degrees, percentages).

(6) "A platform angle larger than $120^{\circ}$ has been recognized as one of the distinguishing features of anvil chipping. Our data show that the average platform angle of the 103 flakes observed is $105^{\circ}(\mathrm{s} d=12.5)$, with a range between $71^{\circ}$ and $133^{\circ}$. None of the platforms observed have an angle in excess of $140^{\circ}$, and about half of the platform angles are between $100^{\circ}$ and $120^{\circ}(n=55)$. " 
entre l'angle d'éclatement et la longueur maximale des éclats lors du débitage sur percuteur dormant : « (...) nous avons mis en relation graphiquement l'angle d'éclatement et la longueur maximale des éclats et avons obtenu une corrélation très faible de $r=0,1305$. Ceci implique que l'angle d'éclatement n'augmente pas nécessairement en fonction de la taille de l'éclat »(Shen et Wang 2000, p. 90)7. Les auteurs concluent que « le débitage sur percuteur dormant produit des éclats qui individuellement présentent peu de différences avec ceux produits par percussion au percuteur dur » (Shen et Wang 2000, p. 95) ${ }^{8}$.

La pratique expérimentale de la percussion sur percuteur dormant nous a également permis une observation intéressante : le nucléus venant frapper le percuteur dormant tangentiellement, selon une trajectoire circulaire, il est impossible de détacher un éclat avec une percussion rentrante (fig. 5 et 6). De ce fait, l'angle entre surface de plan de frappe et surface débitée doit toujours être relativement fermé. L'une des conséquences de ces contraintes d'ordre géométrique est que l'utilisation d'un négatif d'enlèvement comme plan de frappe pour le détachement d'un nouvel éclat ne peut en aucun cas aboutir au détachement d'un grand éclat (fig. 6b). Seule une percussion rentrante permettrait de détacher un grand support mais celle-ci est impossible (fig. 6c). La percussion sur percuteur dormant est donc limitée à des détachements unifaciaux. Par ail-

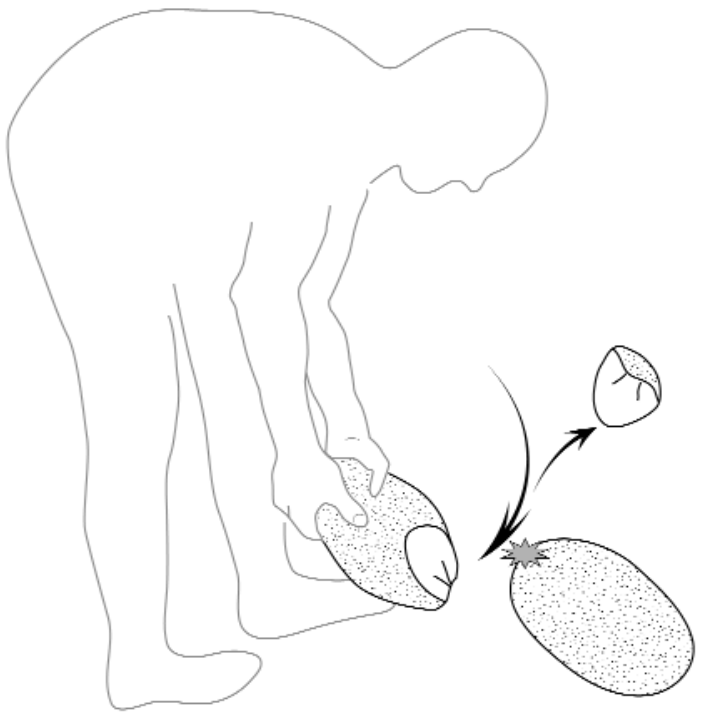

Figure 5 - Percussion sur percuteur dormant.

Figure 5 - Flaking on resting hammer. leurs, des difficultés de préhension apparaissent rapidement si la surface de plans de frappes n'est pas localisée mais s'étend à plus d'un tiers du pourtour du nucléus. La technique impose donc des séries récurrentes d'éclats unifaciaux et unipolaires ou légèrement convergents. Toute production bifaciale et/ou centripète est exclue. De même, il est quasiment impossible d'obtenir de grands éclats à talon non cortical par débitage sur percuteur dormant.

\section{AUTRES DONNÉES ARCHÉOLOGIQUES}

Une revue documentaire des sites acheuléens ayant livré des nucléus à grands éclats permet de recenser d'autres arguments plaidant contre l'utilisation exclusive de la percussion sur percuteur dormant.
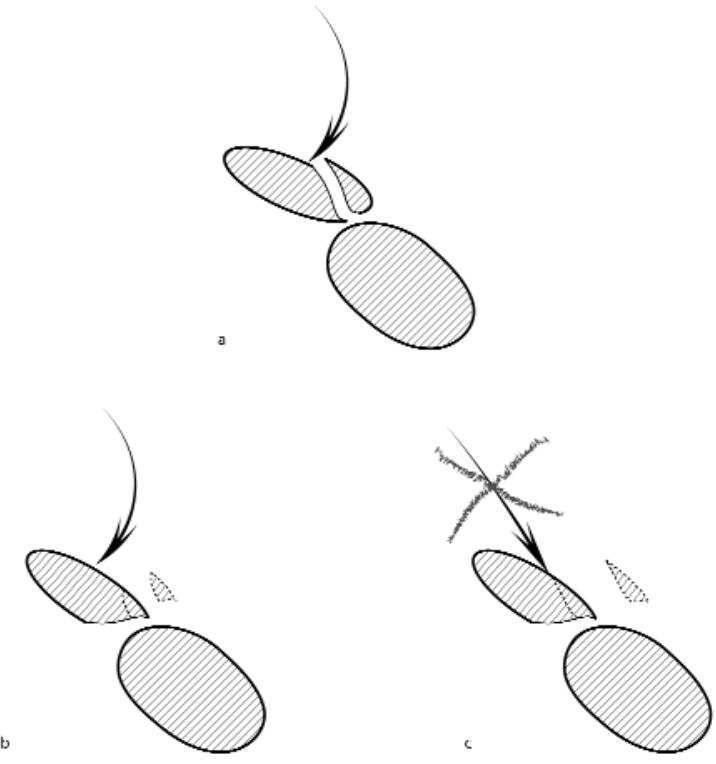

Figure 6 - Coupe théorique du percuteur, du nucléus et de l'éclat produit par percussion sur percuteur dormant. a : détachement d'un grand éclat à partir d'un plan de frappe naturel ; $b$ : résultat obtenu après retournement $d u$ nucléus et percussion selon une trajectoire tangentielle; $c$ : trajectoire rentrante nécessaire pour obtenir un grand éclat après retournement du nucléus ; celle-ci ne peut être mise en œuvre dans la pratique.

Figure 6 - Theoretical cross-section of a hammer, a core and the resulting flake when flaking on resting core. a : flaking of a large flake from a natural striking platform; $b$ : result obtained when turning the core over and pursuing the flaking with a tangential percussion motion; $c$ : internal trajectory necessary to produce a large flake after turning the core over; this trajectory cannot be implemented in practice.

(7) « In order to ascertain whether there is a correlation between the platform angle and the maximum flake length in the anvil-chipping reduction mode, we plotted the platform angle against maximum flake length and obtained a very low correlation of $r=0.1305$. This implies that platform angle is not necessarily increased by increasing the size of the flake. "

(8) - " the anvil-chipping technique produces flakes that individually possess few differences from those produced by the hard-hammer method. » 
Les premiers arguments tiennent aux dimensions et aux caractéristiques volumétriques des nucléus. Outre ceux de Lanne-Darré, des nucléus très massifs ont été recueillis à El Sartalejo en Espagne. Réalisés aux dépens de galets de quartzite, ils dépassent pour la plupart $10 \mathrm{~kg}$ et mesurent plus de $200 \mathrm{~mm}$. Ils ont livré des éclats également imposants, atteignant $180 \times 150 \mathrm{~mm}$. «Leurs dimensions (...) suggèrent qu'ils furent taillés immobilisés au sol, frappés avec un percuteur mobile de poids adéquat, manié des deux mains; le grand poids de ces nodules interdirait toute

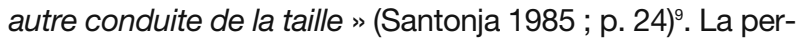
cussion directe au percuteur dur de nucléus immobilisés au sol semble effectivement être la technique la plus probable pour le débitage des grands éclats d'El Sartalejo : en effet, certains nucléus présentent plusieurs négatifs de grands supports extraits de manière centripète, aux dépens d'une surface de débitage préférentielle, sans préparation des surfaces de plan de frappe. Ces négatifs sont parfois utilisés par la suite comme plans de frappe pour produire d'autres grands supports aux dépens de l'autre surface du nucléus (Mourre 2003) (fig. 7). Une telle organisation des enlèvements rend improbable une percussion sur percuteur dormant dans la mesure où la présence de bords tranchants rend leur préhension extrêmement malaisée, voire périlleuse, et où cette technique impose une percussion tangentielle.

Les fouilles conduites par N. Goren-Inbar à Gesher Benot Ya'aqov en Galilée ont également livré des nucléus très volumineux en basalte. L'un d'entre eux était associé à un crâne d'éléphant (Palaeoloxodon antiquus) et aurait été utilisé pour empêcher celui-ci de s'enfoncer dans le sédiment alors qu'il était percuté pour en extraire la cervelle (Goren-Inbar et al. 1994). Ce nucléus est susceptible d'avoir fourni des supports pour les grandes pièces bifaciales ; de grandes dimensions (32 × $26 \times 17 \mathrm{~cm} ; 16,5 \mathrm{~kg}$ ), il évoque un débitage Discoïde avec hiérarchisation des surfaces (surface de plan de frappe/surface de débitage). Le plus grand négatif de la surface de débitage mesure 137 × $104 \mathrm{~mm}$ (Goren-Inbar et al. 1994). Les auteurs signalent d'autres nucléus de dimensions similaires et estiment que leur masse est incompatible avec un débitage sur percuteur dormant (Goren-Inbar et Saragusti 1996).

D'autres arguments sont liés aux méthodes de débitage : en effet, certains grands éclats acheuléens ont été produits par des méthodes impliquant une haute précision de l'impact. Tel est par exemple le cas des hachereaux obtenus par la méthode dite de Tabelbala-Tachenghit (fig. 8). Cette méthode a été successivement décrite par J. Tixier (1956), par B. Champault (1966) puis par J. Zuate y Zuber (In Alimen 1978). Elle est caractérisée par une très forte prédétermination, fruit d'une configuration très précise du nucléus. Le détachement de l'éclat-support correspond pratiquement à l'achèvement du hachereau, les retouches postérieures au débitage étant rares ou limitées. La percussion permettant le détachement de l'éclat-support intervient systématiquement à quelques millimètres d'un dièdre aménagé sur la surface de plans de frappe et se fait selon une direction sud-ouest par rapport au futur hachereau $(91,2 \%$ des cas pour ceux de la collection Champault conservés au Musée de l'Homme) (Mourre 2003). II est hautement improbable qu'une percussion aussi précise ait été réalisée sur percuteur dormant, cette dernière interdisant tout contrôle visuel direct du point d'impact.

Enfin, certains exemples archéologiques plaident en faveur du débitage de grands éclats aux dépens de blocs à l'affleurement, présentant une masse importante leur conférant une inertie suffisante pour demeurer stables lors de la mise en œuvre de percussions relativement violentes. Tel est le cas des éclats de phonolite utilisés comme supports de hachereaux dans le site d'Isenya au Kenya (Mourre 2003). L'étude technologique de ces outils montre que les supports ont été produits par exploitation unipolaire de blocs dormants : en effet, le support et l'enlèvement prédéterminant du tranchant ont presque toujours été détachés dans la même direction.

\section{CONCLUSIONS : QUELLE(S) TECHNIQUE(S) POUR LE DÉBITAGE DE GRANDS ÉCLATS À L'ACHEULÉEN ?}

La percussion sur percuteur dormant implique des nucléus manipulables et donc de masse limitée. Elle implique également une percussion tangentielle avec une trajectoire circulaire. Elle interdit les percussions rentrantes et les enlèvements bifaciaux. Elle suppose donc des séries récurrentes d'éclats unipolaires et unifaciales. Contrairement à un point de vue largement répandu, les angles d'éclatement des produits débités sur percuteur dormant ne sont pas significativement supérieurs à ceux obtenus par percussion directe classique. La percussion sur percuteur dormant ne semble donc pas présenter de stigmate spécifique ou de caractère diagnostique et est sans doute beaucoup moins fréquente que ce qui a été dit. Son utilisation peut être relativisée, voire exclue dans de nombreux sites acheuléens.

La percussion directe classique d'un nucléus mobile à l'aide d'un percuteur tenu en main est probable dans le cas des nucléus de masse limitée et impliquant une haute précision de l'impact (Tabelbala). Elle est en revanche impossible si le nucléus est trop massif. La percussion directe d'un bloc au sol implique l'emploi de percuteurs massifs, une immobilisation ou un calage du nucléus (Lanne Darré, El Sartalejo, Gesher Benot Yaqov) ou bien le débitage de blocs suffisamment importants pour opposer une inertie propre à l'impact (Isenya). N. Toth évoque cette technique à propos de la production des grands éclats ayant servi de support aux outils de l'Acheuléen final de Kalambo Falls en Zambie : "L'expérimentation a démontré qu'en soulevant un lourd percuteur de pierre au-dessus de sa tête et en le projetant des deux mains sur le nucléus, on générait beau- 

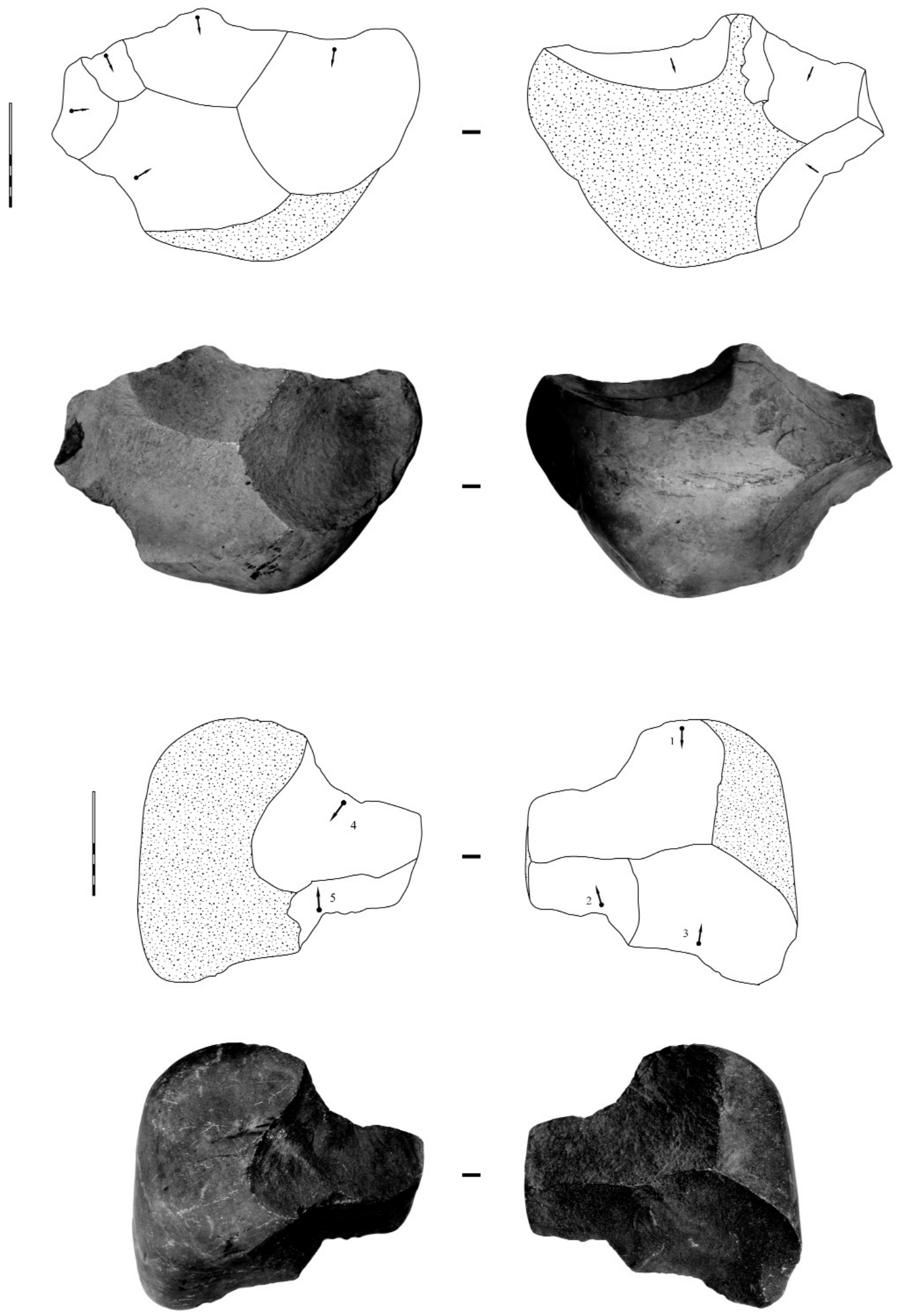

Figure 7 - Nucléus à grands éclats en quartzite d'El Sartalejo, Espagne (clichés et DAO V.M.).

Figure 7 - Quartzite large flakes cores from EI Sartalejo site, Spain (photos and CAD by V.M.) 

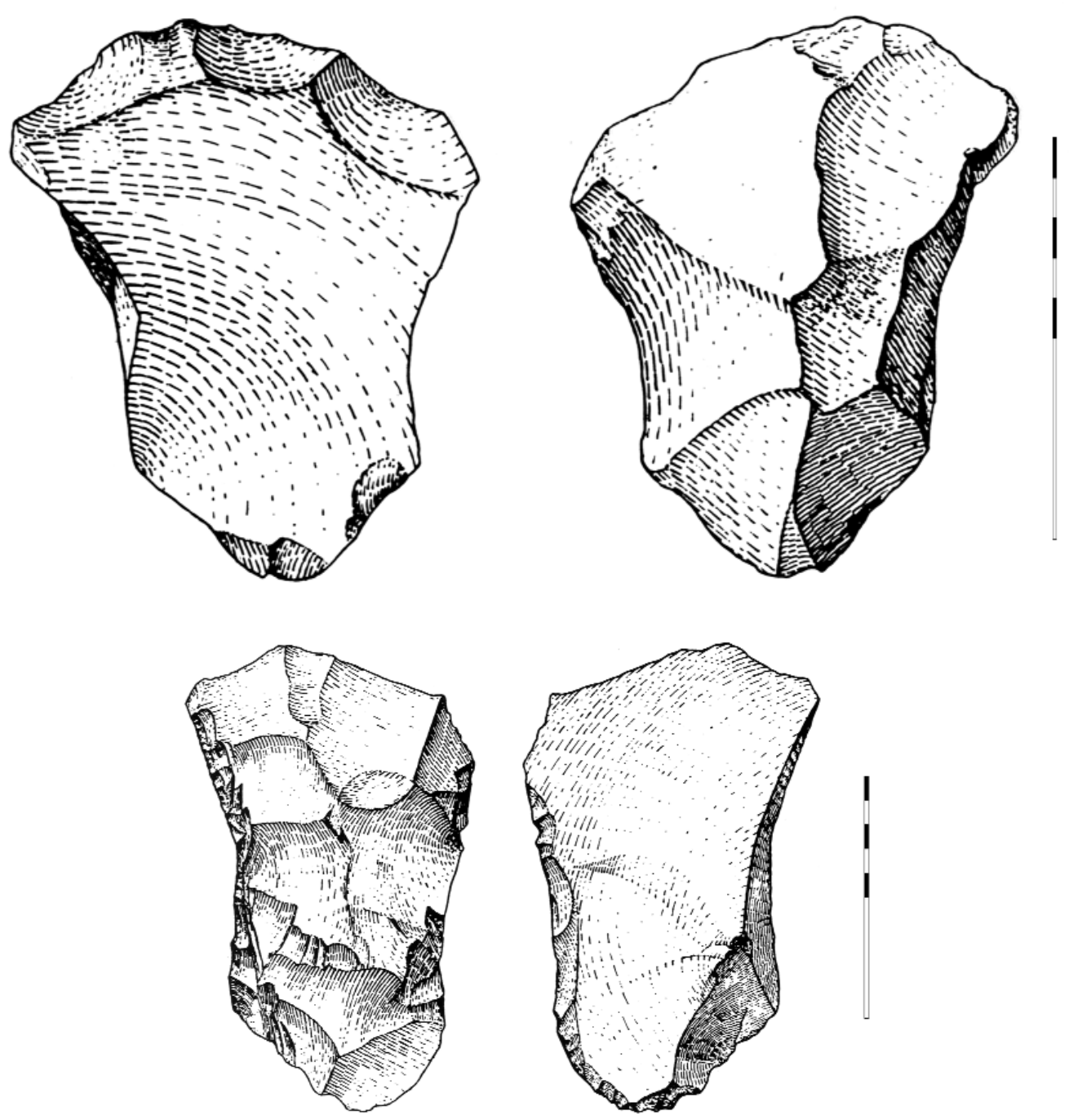

Figure 8 - Nucléus (a) et hachereau (b) de type Tabelbala-Tachenghit du site éponyme (d'après Champault 1966).

Figure 8 - Cores (a) and cleavers (b) of Tabelbala-Tachenghit type from the eponymous site (after Champault 1966).

coup plus de force d'impact qu'en utilisant un percuteur tenu en main. Même si cette technique par projection est habituellement moins précise que la percussion à la main, elle permet de produire de beaucoup plus grands éclats (plus grande dimension parfois supérieure à $40 \mathrm{~cm}$ ) et elle écarte le tailleur de la zone de percussion, réduisant les risques de blessures sévères dues à un doigt piégé entre le percuteur et le nucléus ou à une coupure par l'un des grands éclats détachés. 》 (Toth 2001, p. 602) ${ }^{10}$.

L'emploi de différentes techniques au cours de l'Acheuléen traduit une maîtrise croissante de la gestion des volumes et donc une prédétermination de plus en plus élevée
(Mourre 2003, 2006). Celle-ci est perceptible lorsque l'on analyse les relations entre la direction de débitage des éclats-supports de hachereaux et la direction de détachement des éclats prédéterminant leur tranchant : ces deux directions sont identiques pour les hachereaux les plus anciens (Isenya) alors qu'elles sont opposées pour les hachereaux les plus récents (Tabelbala-Tachenghit). L'évolution des techniques à l'Acheuléen traduit donc une maîtrise croissante de la gestion des volumes.

Même si le comparatisme entre types humains distincts est discutable, les travaux dirigés par P. et A.-M. Pétrequin en Irian Jaya ont démontré que des artisans tailleurs anatomi-

(10) "Experimentation has shown that lifting a heavy stone hammer over one's head and throwing it down with both hands against the core generates much more force of impact than using a hand-held hammerstone. Although this throwing technique normally compromises accuracy relative to hand-held percussion, it can produce much larger flakes (sometimes over $40 \mathrm{~cm}$ in maximum dimension) and removes the knapper from the area of percussion, reducing the chance of serious injury by catching a finger between the hammer and the core or being cut as a large flake is detached. ” 
quement modernes pouvaient manier avec adresse et efficacité des percuteurs de 20 à $30 \mathrm{~kg}$, en l'occurrence pour détacher des supports destinés à la fabrication de haches et d'herminettes polies (Pétrequin et Pétrequin 1993). Selon un principe de parcimonie des hypothèses, il ne nous semble pas pertinent d'envisager pour l'Acheuléen l'utilisation de mécanismes complexes de type balancier ou le recours systématique à la percussion sur percuteur dormant pour la production de grands éclats. En définitive, celle-ci a pu s'effectuer le plus souvent au moyen d'une technique plus classique de percussion de nucléus posés ou immobilisés au sol.

\section{BIBLIOGRAPHIE}

ALIMEN M.-H. 1963 - Enclumes (percuteurs dormants) associés à l'Acheuléen supérieur de l'Ougartien (Oued Farès, Sahara occidental). Bulletin de la Société Préhistorique Française, t. LX, p. 43-47.

ALIMEN M.-H. 1978 - L'évolution de l'Acheuléen au Sahara Nord-Occidental (Saoura - Ougarta - Tabelbala). avec la coll. de J. Zuate y Zuber, Meudon: CNRS, 596 p.

ASHTON N., COOK J., LEWIS S. G. et ROSE J. 1992 High Lodge - Excavation by G. de G. Sieveking 1962-68 and J. Cook 1988. Londres: British Museum Press, 192 p.

BALOUT L. 1967 - Procédés d'analyse et questions de terminologie dans l'étude des ensembles industriels du Paléolithique inférieur en Afrique du Nord. In: Bishop, W.W. et Clark, J. D. (dir.), Background to evolution in Africa, Chicago, p. 701-735.

BAYLE DES HERMENS R. D. 1975 - Recherches préhistoriques en République centrafricaine. Nanterre : Labethno, Publications du Laboratoire d'Ethnologie et de sociologie comparative, Université de Paris X, 343 p.

BERTOUILLE H. 1989 - Théories physiques et mathématiques de la taille des outils préhistoriques. Cahiers du Quaternaire $n^{\circ} 15$, CNRS éd., $100 \mathrm{p}$.

BORDES F. 1947 - Étude comparative des différentes techniques de taille du silex et des roches dures. L'Anthropologie, t. 51, p. 1-29.

BORDES F. 1961 - Typologie du Paléolithique ancien et moyen. Bordeaux: Delmas, Publications de l'Institut de Préhistoire de l'Université de Bordeaux, Mémoire $n^{\circ} 1$, $111 \mathrm{p}$.

BORDES F. 1984 - Leçons sur le Paléolithique - 3. Le Paléolithique hors d'Europe. Cahiers du Quaternaire $n^{\circ} 7$, Ed. du CNRS, 3 t., 352 p.

BREUIL H. 1949a - Division du travail au Paléolithique en Afrique du Sud. Bulletin de la Société Préhistorique Française, t. XLVI, n 7-8, p. 243-244.

BREUIL H. 1949b - Beyond the bounds of History - Scenes from the Old Stone Age. London: P.R. Gawthorn, Ltd, $100 \mathrm{p}$.
BREUIL H. 1954 - Prolégomènes à une classification préhistorique - Discours du Président entrant. Bulletin de la Société Préhistorique Française, t. LI, fasc. 1-2, p. 7-15.

BREUIL H. et LANTIER R. 1951 - Les Hommes de la pierre ancienne - Paléolithique et Mésolithique. Paris: Payot, $335 \mathrm{p}$.

CAHEN D. 1975 - Le site archéologique de La Kamoa (région de Shaba, rép. du Zaïre) de l'Age de Pierre ancien à l'Age du fer. Tervuren: Musée royal de l'Afrique centrale Tervuren (Belgique) - Annales, série in $8^{\circ}$ - Sciences humaines, 365 p. + fig.

CAPDEVILLE J.-P., CHALARD P., JARRY M., MILLET D. et O'YL W. 1997 - Le gisement acheuléen d'En Jacca - La Sauvegarde à Colomiers (Haute-Garonne) : nouvelles données. Paléo, n 9, p. 69-99.

CHAMPAULT B. 1966 - L'Acheuléen évolué au Sahara occidental - Notes sur l'homme au Paléolithique ancien. Thèse de doctorat soutenue le 17 nov. 1966. Faculté de Lettres, Paris, 2 vol., 1068 p.

COLONGE D. 2005 - Économie des matières premières lithiques sur un site acheuléen du piémont pyrénéen : Lanne-Darré à Uglas (Hautes-Pyrénées). In: Jaubert, J. et Barbaza, M. (dir.), Territoires, déplacements, mobilité, échanges pendant la préhistoire. Terres et hommes du Sud, Actes du 126e congrès du CTHS, Toulouse, 2001, p. 33-48.

COLONGE D., LELOUVIER L.-A. et MOURRE V. à paraître - Approvisionnement et économies des matières premières dans l'Acheuléen du piémont occidental des Pyrénées françaises. In: Marchand, G. et Querré, G. (dir.), Roches et Sociétés de la Préhistoire, actes du colloque de Rennes, 28-30 avril 2010

COLONGE D. et MOURRE V. 2009 - Quartzite et quartzites : aspects pétrographiques, économiques et technologiques des matériaux majoritaires du Paléolithique ancien et moyen du Sud-Ouest de la France. In: Grimaldi, S. et Cura, S. (dir.), Technological analysis on quartzite exploitation, XVème Congrès de I'UISPP, 4-9 septembre 2006, Lisbonne, vol. 39, session WS 15, BAR International Series 1998, p. 3-12.

COLONGE D. et TEXIER J.-P. 2005 - Le gisement acheuléen de Lanne-Darré (Uglas, Hautes-Pyrénées) et perspectives régionales dans le Sud-Ouest de l'Europe. In: Données récentes sur les modalités de peuplement et sur le cadre chronostratigraphique, géologique et paléogéographique des industries du Paléolithique inférieur et moyen en Europe, BAR International Series 1364, Actes du Colloque International de Rennes, 22-25 septembre 2003, p. 203-214.

COYE N. D. 2006 - Sur les chemins de la préhistoire : l'abbé Breuil du Périgord à l'Afrique du Sud. Paris: Somogy Éditions d'Art, 223 p. 
CRABTREE D. E. 1972 - An introduction to flintworking. Occasional Papers of the Idaho state University Museum, Pocatello, Idaho, $n^{\circ} 28,98$.

FORESTIER H. 1993 - Le Clactonien : mise en application d'une nouvelle méthode de débitage s'inscrivant dans la variabilité des systèmes de production lithique du Paléolithique ancien. Paléo, n 5, p. 53-82.

GILEAD D. 1973 - Cleavers in Early Palaeolithic Industries of Israel. Paléorient, vol. I, p. 73-86.

GOREN-INBAR N., LISTER A., WERKER E. et CHECH M. 1994 - A butchered elephant skull and associated artifacts from the Acheulian site of Gesher Benot Ya'aqov, Israel. Paléorient, vol. 20, 1, p. 99-112.

GOREN-INBAR N. et SARAGUSTI I. 1996 - An Acheulian biface assemblage from Gesher Benot Ya'aqov, Israel : indications of african affinities. Journal of Field Archaeology, vol. 23, $\mathrm{n}^{\circ} 1$, p. 15-30.

HEINZELIN DE BRAUCOURT J. D. 1962 - Manuel de typologie des industries lithiques. Bruxelles: Institut royal des Sciences naturelles de Belgique, 74 p., 50 fig.

KLEINDIENST M. R. 1961 - Variability within the late Acheulian assemblage in Eastern Africa. South African Archaeological Bulletin, 16, p. 35-52.

LEAKEY M. D. 1971 - Olduvai Gorge - vol. 3 : Excavations in Beds I and II, 1960-1963. Cambridge: Cambridge University Press, $306 \mathrm{p}$.

LEAKEY M. D. 1975 - Cultural patterns in the Olduvai sequence. In: Butzer, K. W. et Isaac, G. L. (dir.), After the Australopithecines, Mouton, p. 477-493.

MOURRE V. 2003 - Implications culturelles de la technologie des hachereaux. Thèse de Doctorat. Université de Paris X - Nanterre, 3 vol., 880 p.

MOURRE V. 2006 - Émergence et évolution de la prédétermination au Paléolithique. In: Astruc, L., Bon, F., Léa, V., Milcent, P.-Y. et Philibert, S. (dir.), Normes techniques et pratiques sociales : " de la simplicité des outillages pré- et protohistoriques " XXVle Rencontres Internationales d'Archéologie et d'Histoire d'Antibes, 20 - 22 octobre 2005, Antibes-Juan-les-Pins, p. 61-74.
MUYA WA BITANKO KAMUANGA D. 1991 - Zaïre - Âge de la Pierre Ancien. In: Lanfranchi, R. et Clist, B. (dir.), Aux origines de l'Afrique centrale, Centres Culturels Français d'Afrique centrale, Centre International des Civilisations Bantu, Ministère de la Coopération et du Développement, Centre Culturel Français de Libreville, Sépia, p. 75-81.

PEI W.-C. 1936 - Le rôle des phénomènes naturels dans l'éclatement et le façonnement des roches dures utilisées par l'Homme préhistorique. Thèse de Doctorat. Institut de Paléontologie Humaine, $75 \mathrm{p}$.

PÉTREQUIN P. et PÉTREQUIN A.-M. 1993 - Écologie d'un outil : la hache de pierre en Irian Jaya (Indonésie). Paris : CNRS Éditions, Monographie du CRA 12, 469 p.

SANTONJA M. 1985 - El yacimiento achelense de El Sartalejo (valle del Alagón, Cáceres). Estudio preliminar. Series de Arqueologia Extremeña, 2, Univ. de Extremadura, Cáceres, p. 1-109.

SHEN C. et WANG S. 2000 - A preliminary study of the anvil-chipping technique : experiments and evaluations. Lithic technology, vol. 25, $n^{\circ}$ 2, p. 81-100.

STEKELIS M. 1960 - The Palaeolithic deposits of Jisr Banat Yaqub. The Bulletin of the Research Council of Israël, section G. Geo-Sciences, vol. 9G, n²-3, p. 61-90.

TIXIER J. 1956 - Le hachereau dans l'Acheuléen nord-africain - Notes typologiques. In : Congrès préhistorique de France - Compte-rendu de la XVème session - PoitiersAngoulême - 15-22 juillet 1956, p. 914-923.

TOTH N. 2001 - Experiments in quarrying large flake blanks at Kalambo Falls. In: Clark, J. D. (dir.), Kalambo Falls Prehistoric Site - Volume 3, The Earlier Cultures: Middle and Earlier Stone Age, Cambridge: Cambridge University Press, p. 600-604.

TUFFREAU A. (dir.) 1996 - L'Acheuléen dans l'Ouest de l'Europe. Lille: Actes du Colloque de Saint Riquier, 610 juin 1989, Publications du CERP, $n^{\circ} 4$, Université des Sciences et Technologies de Lille, 7-8.

VAN RIET LOWE C. 1951 - A new african Acheul stage IV site in Tanganyika. South African Archaeological Bulletin, vol. $6, n^{\circ} 24$, p. 94-98. 